\title{
Research on the Grey Verhulst Model Based on Particle Swarm Optimization and Markov Chain to Predict the Settlement of High Fill Subgrade in Xiangli Expressway
}

\author{
Haiming Liu $\mathbb{D},{ }^{1}$ Wei Guo $\mathbb{D}{ }^{1},{ }^{1}$ Chao Zhang $\mathbb{D}{ }^{2}$, and Huaihao Yang $\mathbb{D}^{1}$ \\ ${ }^{1}$ Yunnan Key Laboratory of Disaster Reduction in Civil Engineering, Faculty of Civil Engineering and Mechanics, \\ Kunming University of Science and Technology, Kunming 650500, China \\ ${ }^{2}$ State Key Laboratory of Geomechanics and Geotechnical Engineering, Institute of Rock and Soil Mechanics, \\ Chinese Academy of Sciences, Wuhan 430071, China
}

Correspondence should be addressed to Haiming Liu; haiming0871@163.com

Received 23 December 2018; Revised 14 March 2019; Accepted 7 April 2019; Published 17 April 2019

Academic Editor: Marko Canadija

Copyright (C) 2019 Haiming Liu et al. This is an open access article distributed under the Creative Commons Attribution License, which permits unrestricted use, distribution, and reproduction in any medium, provided the original work is properly cited.

It is of vital significance to accurately forecast the settlement of high fill subgrade, which is the foundation for disaster prevention and treatment of subgrade. According to the monitoring data of high fill subgrade, a novel model, called PSOMGVM model, based on particle swarm optimization (PSO) and Markov chain is proposed. Firstly, the typical characteristics of settlement curve are analyzed from the aspect of geomechanics theory and based on the grey theory, the grey Verhulst model (GVM) with unequal time-interval is proposed. Then, according to the theory of Markov chain, the grey Verhulst model is built to revise the relative residuals of the GVM, in which the effects of volatility characteristics can be considered. Finally, the PSOMGVM model based on PSO algorithm and Markov chain is set up, which whitens the parameters of the grey interval. In order to demonstrate the fitness and the ability of the proposed model, five competing models are introduced to predict the settlement of the high fill subgrade of Xiangli Expressway in Yunnan Province. Through the analysis of APE, MAPE, and RMSE, it states that the accuracy and performance of the PSOMGVM model outperform the other five competing models for simulative and predictive periods.

\section{Introduction}

Along with the continuous promotion of "the Belt and Road," the expressway of Yunnan Province, which is the bridgehead in Southeast Asia, will usher in a new development opportunity. The complex geological environment and high mountain landform of Yunnan district have resulted in a large number of high fill subgrade [1]. The settlement control of high fill subgrade is vitally important for the safety and operation of the expressway, which is the premise and key for predicting the settlement of high fill subgrade accurately. At present, the prognostic methods of settlement can be divided as follows. (1) The prognostic method is based on the theory of soil mechanics, which includes the layer-wise summation method [2], the finite element method [3], the empirical equation method [4] and so on. Generally, the geometric condition, boundary condition, or constitutive model of the above methods is inconsistent with the engineering practice, which will affect the accuracy of prediction results [5]. (2) The prognostic method is based on the monitoring data, which includes the Asaoka method [6], hyperbola method [7], three-point method [8], settlement difference method [9], genetic algorithm [10], neural network method [11], grey theory method [12], and so on. This kind of method has attracted the attention of many scholars and is widely used in engineering practice at home and abroad.

At present, many scholars have carried out relevant research on prognostic model based on the grey theory and have obtained some beneficial results and valuable achievements. An optimized grey discrete Verhulst model is proposed to predict the settlement of the foundation pit by Zhang et al. [13]. A modified grey Verhulst model method using Fourier series of error residuals is proposed by Ming et al. [14]. A flexible generalization of the grey Verhulst model is 
put forward by Evans [15]. A grey Verhulst sliding mode controller is proposed for the laboratory antilock braking system by Boopathi et al. [16]. An improved version of Saito's method together with the grey Verhulst model is presented by Miao et al. [17]. A grey time-delayed Verhulst model used the method of grey differential equations is established by Wang et al. [18]. Wang et al. present a method for physical software time stage-effort prediction based on the grey models $\operatorname{GM}(1,1)$ and Verhulst [19]. The grey Verhulst model based on PSO algorithm is adopted to discuss the relationship between $\mathrm{CO}_{2}$ emissions and economy by Wang and Li [20]. The grey Verhulst model and inverse Verhulst model are established to predict the mechanical behavior of a minicomposite by Wang et al. [21]. An optimal grey Verhulst model is proposed to predict the concentration of nitrogen in seawater by Hu et al. [22]. The Verhulst model and $\operatorname{DGM}(1,1)$ model are employed to simulate the development tendency based on the information decomposing and model combination by Zeng et al. [23]. However, the data fluctuation is not taken into consideration in these papers.

Based on the grey theory, the grey Verhulst model (GVM) with unequal time-interval is proposed in this paper. Combined with the Markov chain theory, the relative residual sequence of GVM model is predicted. On this basis, the grey Verhulst model (MGVM) based on the Markov chain is established, which can revise the relative residual error of GVM model. Then, the grey Verhulst model based on PSO algorithm and Markov chain (PSOMGVM) is established by whitening Markov state interval parameters. The settlement monitoring data of high fill subgrade of Xiangli Expressway in Yunnan Province is took as an example, and the feasibility of PSOMGVM prognostic model is verified by the comparing and analyzing of different prognostic models.

\section{Analyze the Characteristic of "S" Shape Curve during Settlement}

According to the theory of soil mechanics, the settlement of soil can be divided into three parts: the instantaneous settlement, the primary consolidation settlement, and the secondary consolidation settlement [24]. The instantaneous settlement occurs at the moment of the imposing of force, which is caused by the shear strain under the condition of constant volume. Bjerrum pointed out that this settlement is lateral yielding [25]. It shows that the relationship curve between the settlement $s$ and the time $t$ does not pass through the origin, as shown in point $a$ of Figure 1. The primary consolidation settlement and the secondary consolidation settlement vary with the load and the time, which is similar to the growth curve described by Verhulst model. A large number of the engineering practices and the monitoring results of land subsidence with long-term reveal the characteristic of "S" shape curve $[26,27]$. In fact, the growth curve reflects the process of the occurrence, development, maturity, and ultimate state of the settlement. The characteristic of " $S$ " shape can be divided into four stages, which are shown in Figure 1.

(1) In the elastic stage $a b$, the settlement linearly increases with time. At the initial stage of the loading, the soil is in the

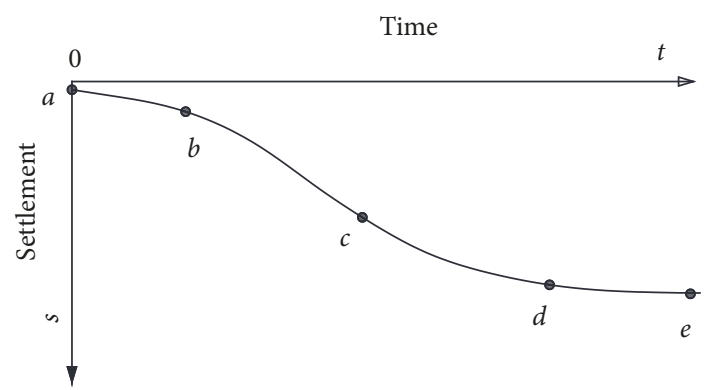

FIgURE 1: The typical settlement curve of foundation.

state of elastic stress. Then, the settlement increases almost linearly with the load.

(2) In the elastoplastic development stage $b c$, the settlement rate increases with time. With the increase of load, the plastic zone develops continuously. When the soil stress is increasing, the settlement and its rate are increasing [28].

(3) In the elastoplastic mature stage $c d$, the settlement rate decreases with the time. Because the consolidation is not completed and the soil has the characteristics of rheology, the soil settlement will increase with time, but the settlement rate will decrease [29].

(4) In the stable stage de, the settlement tends to be stable. In theory, when the time is infinite, the settlement reaches its limit state.

Based on the above analysis, the relationship curve between the settlement $s$ and time $t$ is "S" shape curve without passing the origin in the whole settlement process.

\section{Methodological Framework}

\subsection{The Grey Verhulst Model with Unequal Time-Interval}

3.1.1. The Data Conversion with Unequal Time-Interval. Assume that the original data sequence is

$$
Y=\left\{y\left(t_{1}\right), \cdots, y\left(t_{k}\right), \cdots, y\left(t_{n}\right)\right\}, \quad y\left(t_{k}\right) \geq 0
$$

Assuming $\Delta t_{i}=t_{i+1}-t_{i}, \Delta t_{j}=t_{j+1}-t_{j}$, if $i \neq j$, there is at least one or more time-intervals which satisfy the condition $\Delta t_{i} \neq \Delta t_{j}, \quad i, j \in\{1,2, \cdots, n-1\}$. This data sequence is called the unequal time-interval data sequence.

For the raw sequence with unequal time-interval, the following methods can be used to convert them into the data sequence with equal time-interval.

(1) Calculate the average time-interval

$$
m=\sum_{i=1}^{n-1} \Delta t_{i}=\frac{1}{n-1}\left(t_{n}-t_{1}\right)
$$

(2) Calculate the interpolation coefficient of time point $t_{i}$

$$
\mu_{i}=(i-1) m-\left(t_{i}-t_{1}\right), \quad i=1, \cdots, n
$$


(3) Generate the data sequence $X^{(0)}$ with equal timeinterval

$$
x^{(0)}(i)=y\left(t_{i}\right)+\frac{\mu_{i} y\left(t_{i+1}\right)-\mu_{i} y\left(t_{i}\right)}{\Delta t_{i}}, \quad \begin{aligned}
& \\
& i=1, \cdots, n-1
\end{aligned}
$$

(4) The data sequence with equal time-interval can be obtained as follows:

$$
x^{(0)}=\left(x^{(0)}(1), x^{(0)}(2), \cdots, x^{(0)}(n)\right)
$$

3.1.2. Grey Verhulst Model. Generally, the cumulative generating operator can be obtained by accumulating the original data.

$$
x^{(1)}=\left(x^{(1)}(1), x^{(1)}(2), \cdots, x^{(1)}(n)\right)
$$

where $x^{(1)}(i)=\sum_{k=1}^{i} x^{(0)}(k), i=1,2, \cdots$

The average sequence of its accumulated data sequence is as follows:

$$
Z^{(1)}(k)=x^{(1)}(k)+x^{(1)}(k-1), \quad k=2,3, \cdots, n
$$

The grey Verhulst model is as follows:

$$
x^{(0)}(k)+a z^{(1)}(k)=b\left(z^{(1)}(k)\right)^{2}
$$

The whitening equation of grey Verhulst model is as follows:

$$
\frac{d x^{(1)}}{d t}+a x^{(1)}=b\left(x^{(1)}\right)^{2}
$$

The variation rule of grey Verhulst prognostic model is consistent with the settlement curve in the whole process. It has the characteristic of " $\mathrm{S}$ " shape type, which is similar to the logistic growth model.

The whitening response equation of grey Verhulst model is as follows:

$$
\widehat{x}^{(1)}(k)=\frac{a / b}{1+\left(a / b x^{(0)}(1)-1\right) e^{a(k-1)}}
$$

The prognostic value of $\hat{x}^{(0)}(k+1)$ is obtained by the cumulative subtraction of $\widehat{x}^{(1)}(k+1)$.

$$
\widehat{x}^{(0)}(k)=\widehat{x}^{(1)}(k)-\widehat{x}^{(1)}(k-1)=\frac{a x^{(0)}(1)\left(a-b x^{(0)}(1)\right)\left(1-e^{a}\right) e^{a(k-2)}}{\left(b x^{(0)}(1)+\left(a-b x^{(0)}(1)\right) e^{a(k-1)}\right)\left(b x^{(0)}(1)+\left(a-b x^{(0)}(1)\right) e^{a(k-2)}\right)}
$$

In $(8), x^{(0)}(k)$ and $z^{(1)}(k)$ are substituted by $k=$ $2,3, \cdots, n$.

$$
\begin{gathered}
x^{(0)}(2)+a z^{(1)}(2)=b\left(z^{(1)}(2)\right)^{2} \\
x^{(0)}(3)+a z^{(1)}(3)=b\left(z^{(1)}(3)\right)^{2} \\
\vdots \\
x^{(0)}(n)+a z^{(1)}(n)=b\left(z^{(1)}(n)\right)^{2}
\end{gathered}
$$

It can be obtained by the least square method.

$$
\widehat{\alpha}=(a, b)^{T}=\left(B^{T} B\right)^{-1} B^{T} Y_{n}
$$

where

$$
\begin{aligned}
& Y_{n}=\left[\begin{array}{c}
x^{(0)}(2) \\
x^{(0)}(3) \\
\vdots \\
x^{(0)}(n)
\end{array}\right] \\
& B=\left[\begin{array}{ccc}
-z^{(1)}(2) & \left(z^{(1)}(2)\right)^{2} \\
-z^{(1)} & (3) & \left(z^{(1)}(3)\right)^{2} \\
\vdots & \vdots \\
-z^{(1)} & (n) & \left(z^{(1)}(n)\right)^{2}
\end{array}\right]
\end{aligned}
$$

The obtained $a$ and $b$ are substituted into (11) to get $X^{(1)}$. Then, it is converted into the data sequence with unequal time-interval as before.

$$
\begin{aligned}
& \widehat{x}^{(0)}(i)=\frac{m-\mu_{i}}{m} \widehat{x}^{(0)}(i+1)+\frac{\mu_{i}}{m} \widehat{x}^{(0)}(i), \\
& i=1, \cdots, n-1
\end{aligned}
$$

3.2. The MGVM Model Based on Markov Optimization. Given that the prognostic value is affected by many factors, the relative error of prognostic value is volatile, which can be optimized by Markov theory.

The relative error of the prognostic model is

$$
\begin{aligned}
\varepsilon(i) & =\frac{x^{(0)}(i)-\widehat{x}^{(0)}(i)}{x^{(0)}(i)}, \quad i=1,2, \cdots, n \\
\widehat{x}_{M G V M}^{(0)}(i) & =\frac{\widehat{x}^{(0)}(i)}{1-\varepsilon(i)}, \quad i=1,2, \cdots, n
\end{aligned}
$$

According to $\varepsilon(1), \cdots, \varepsilon(i), \cdots$, the $\varepsilon(n+1)$ can be deduced. If $\varepsilon(n+1)$ can be calculated, the $\widehat{x}_{M G V M}^{(0)}(n+1)$ of (17) can be taken as the prognostic value when $k=n+1$.

Assuming that the data sequence is $x(t)$, the states of the time $t_{1}, \cdots, t_{k}$ are known. If the state of $t_{k+1}$ is only related to the state of $t_{k}$ and it has nothing to do with the previous 
states before $t_{k}$, as shown in (18), this data sequence can be called Markov chain process, which has no aftereffect.

$$
\begin{aligned}
& P\left(\left(Y_{t}=j\right) \mid Y_{t_{1}}=i_{1}, Y_{t_{2}}=i_{2}, \cdots, Y_{t_{n}}=i_{n}\right) \\
& \quad=P\left(\left(Y_{t}=j\right) \mid Y_{t_{n}}=i_{n}\right)
\end{aligned}
$$

The basic procedure of Markov optimization is as follows. The relative residual error of original sequence $\varepsilon(1), \varepsilon(2), \cdots, \varepsilon(n)$ can be segmented into the $l$ state, which is divided by equidistant in general. The variable of state $i$ is denoted as $S_{i}(i=1,2, \ldots, l)$, whose the upper limit is $S_{i}^{u}$ and the lower limit is $S_{i}^{l}$. The state transfer matrix of the residual sequence is obtained according to the Markov chain, and the prognostic value can be revised through (17).

The one-step state of transition probability matrix is

$$
p=\left[\begin{array}{cccc}
p_{11} & p_{12} & \cdots & p_{1 l} \\
p_{21} & p_{22} & \cdots & p_{2 l} \\
\cdots & & \\
p_{n 1} & p_{n 2} & \cdots & p_{n l}
\end{array}\right]
$$

where

$$
P_{i j}=\frac{N_{i j}}{N_{i}} \quad(i, j=1,2, \cdots, l)
$$

Assuming that the state of $k=n$ is

$$
\varepsilon(n)=\left(p_{1}, p_{2}, \cdots, p_{l}\right)
$$

If $\varepsilon(n) \in S_{i}$, then $p_{i}=1$. If $\varepsilon(n) \bar{\epsilon} S_{i}$, then $p_{i}=0, i=$ $1,2, \cdots, l$.

The state of $k=n+1$ is

$$
\varepsilon(n) p=\left(\sum_{i=1}^{l} p_{i} p_{i 1}, \sum_{i=1}^{l} p_{i} p_{i 2}, \cdots, \sum_{i=1}^{l} p_{i} p_{i n}\right)
$$

The probability of the corresponding state is determined according to (21), and the predicted value of the residual error can be calculated.

$$
\varepsilon(n+1)=\sum_{j=1}^{l} \overline{S_{j}} \cdot \sum_{i=1}^{l} p_{i} p_{i j}
$$

Based on Markov optimization, the prognostic value of the MGVM model can be determined.

$$
\widehat{x}_{M G V M}^{0}(k+1)=\widehat{x}^{0}(k+1)+\frac{S_{k+1}^{u}+S_{k+1}^{l}}{2}
$$

3.3. The PSOMGVM Model Based on PSO Algorithm. When the particle swarm composed of $n$ particles is flying at a certain speed in exploring space, the particle will consider its own historical optimum and the historical optimum of all particles. It will constantly update the flight speed and the direction to search for the optimal solution, which is called particle swarm optimization (PSO) algorithm.
The velocity and the position of particles are updated according to (25) and (26).

$$
\begin{aligned}
& v_{k d}^{i+1}=\omega v_{k d}^{i}+c_{1} r_{1}\left(h_{k d}^{i}-x_{k d}^{i}\right)+c_{2} r_{2}\left(h_{g d}^{i}-x_{k d}^{i}\right) \\
& x_{k d}^{i+1}=x_{k d}^{i}+v_{k d}^{i+1}
\end{aligned}
$$

where

$$
\begin{aligned}
& x_{k d}=\left(x_{k 1}, x_{k 2} \cdots, x_{k c}\right) \\
& v_{k d}=\left(v_{k 1}, v_{k 2} \cdots, v_{k c}\right) \\
& h_{k d}=\left(h_{k 1}, h_{k 2} \cdots, h_{k c}\right) \\
& h_{g d}=\left(h_{g 1}, h_{g 2} \cdots, h_{g c}\right)
\end{aligned}
$$

$\omega$ is inertia weighting coefficient, and it can change the search ability of the PSO algorithm. When $\omega=1$, it is called the basic PSO algorithm.

In order to improve the search efficiency of the PSO algorithm, the dynamic inertia weight coefficient $\omega_{i t e r}$, the dynamic cognitive acceleration coefficient $c_{1, i t e r}$, and the dynamic social acceleration coefficient $c_{2, \text { iter }}$ are proposed. When the single coefficients $\omega, c_{1}$, and $c_{2}$ are adopted, it often falls into the "local optimization solution". This strategy can avoid the risk of getting in the "local optimization solution".

$$
\begin{aligned}
& \omega_{\text {iter }}=\omega_{\text {max }}-\frac{\omega_{\text {max }}-\omega_{\text {min }}}{N_{\text {iter }_{\text {max }}}} \times N_{\text {iter }} \\
& c_{1, \text { iter }}=c_{\text {max }}^{1}-\frac{c_{\text {max }}^{1}-c_{\text {min }}^{1}}{N_{\text {iter }} r_{\text {max }}} \times N_{\text {iter }} \\
& c_{2, \text { iter }}=c_{\text {max }}^{2}-\frac{c_{\text {max }}^{2}-c_{\text {min }}^{2}}{N_{\text {iter }_{\text {max }}}} \times N_{\text {iter }}
\end{aligned}
$$

Through the PSO algorithm, the optimization of interval state of the weight coefficient $a_{i}$ can be obtained. The PSOMGVM prognostic value is as follows:

$$
\begin{aligned}
\widehat{x}_{P S O M G V M}^{0}(k+1)= & \widehat{x}^{0}(k+1) \\
& +\left[\alpha_{i} S_{k+1}^{u}+\left(1-\alpha_{i}\right) S_{k+1}^{l}\right]
\end{aligned}
$$

The calculation flow of the PSOMGVM prognostic model based on the PSO algorithm and the Markov chain is shown in Figure 2.

3.4. Inspection the Accuracy of Prediction. In order to appraise the predictive accuracy of the competing models, the first thing is to choose the appropriate quantitative appraise indices, which can effectively judge whether the predictive accuracy is excellent or inaccurate. In this paper, three classical statistics indicators, namely, absolute percentage error $(A P E)$, mean absolute percentage error $(M A P E)$, and root mean squared error ( $R M S E)$, are adopted to elaborate the 


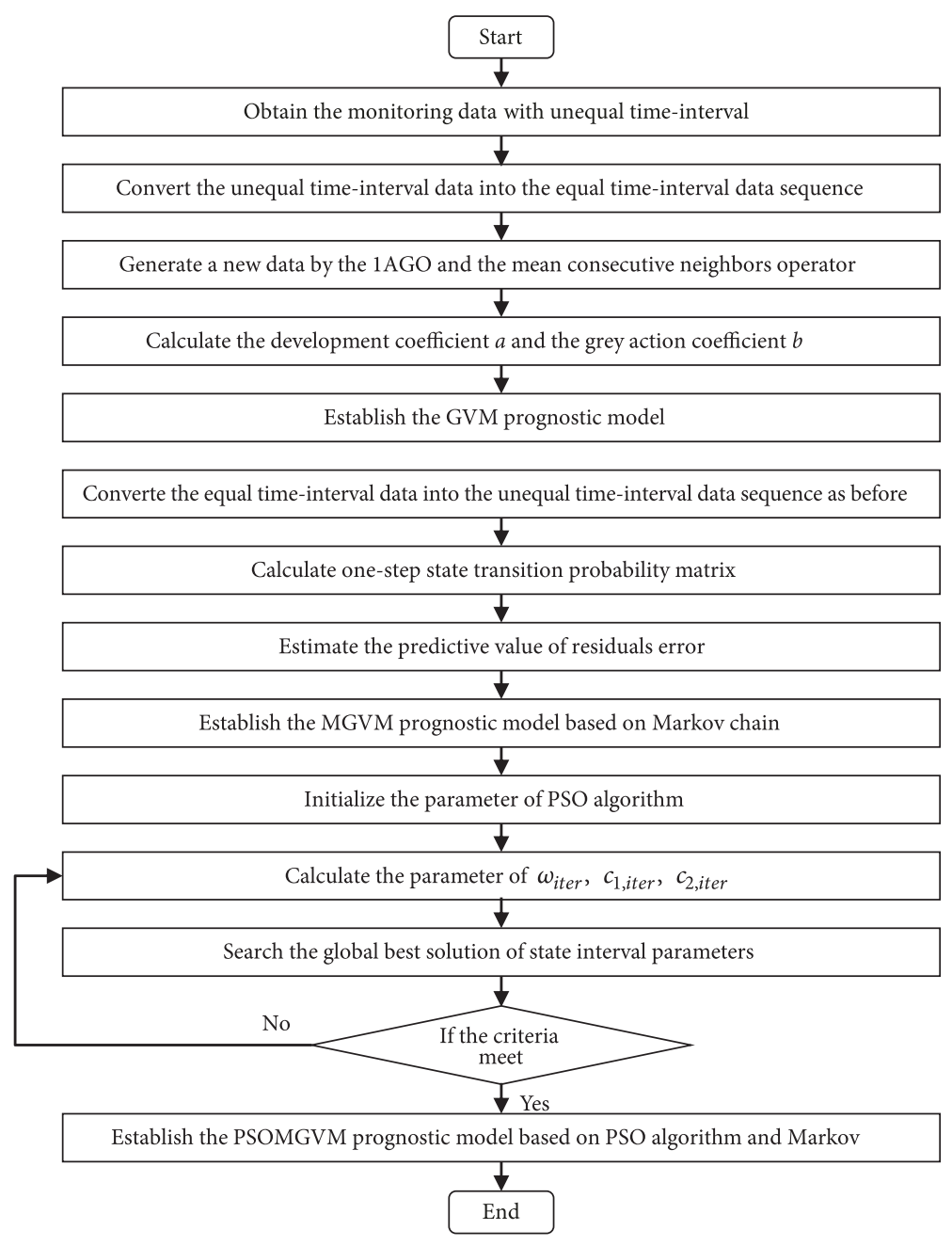

FIgURE 2: The flow-process diagram of the PSOMGVM prognostic model.

predictive performance of different models. The APE, MAPE, and RMSE are calculated as the following three equations:

$$
\begin{aligned}
A P E & =\left|x^{(0)}(i)-x^{(1)}(i)\right|, \quad i=1,2, \cdots, n \\
M A P E & =\frac{1}{n} \sum_{i=1}^{n}\left|\frac{x^{(1)}(i)-x^{(0)}(i)}{x^{(0)}(i)}\right| \\
R M S E & =\sqrt{\frac{1}{n-1} \sum_{i=1}^{n}\left[x^{(0)}\left(t_{i}\right)-\bar{x}^{(0)}\right]^{2}}
\end{aligned}
$$

Generally, the smaller the APE, RMSE, and MAPE values are, the more accuracy the prognostic model is. According to the Lewis theory [30], the predictive performance can be divided into four grades, which is shown in Table 1.

\section{An Example of the Settlement of High Fill Subgrade}

In order to demonstrate the simulative and predictive capabilities of the proposed model in Section 3, three competing
TABLE 1: Appraise criteria of MAPE based on Lewis [unit: \%].

\begin{tabular}{lcccc}
\hline MAPE & $<10$ & $10-20$ & $20-50$ & $>50$ \\
\hline Predictive ability & Excellent & Good & Reasonable & Inaccurate \\
\hline
\end{tabular}

models, namely, the classical GM(1,1), the PSOMGM(1,1) model based on the optimization of Markov chain and classical PSO algorithm, and the ARIMA model, are introduced to elaborate the forecasting performances. The data points from July 2017 to April 2018 are adopted as the input source data to forecast parameters, build competing models, and proofread simulative performance. Meanwhile, the observation data points from April 2018 to June 2018 are employed as the validation data for verifying the predictive accuracy. Through the analysis of evaluation indices of competing models in Section 3.4, the model with the best performance will be used to predict the settlement of high fill subgrade.

4.1. Data Collection. The Xiangli Expressway of Yunnan Province is a section of the Beijing-Tibet expressway of the national network G0613. The Xiangli Expressway has the 
TABLE 2: The unequal data points are converted into the data points with equal time-interval for settlement of high fill subgrade in Xiangli Expressway [unit: $\mathrm{mm}$ ].

\begin{tabular}{|c|c|c|c|c|c|c|c|c|c|c|c|c|c|}
\hline \multirow{2}{*}{ Date } & \multicolumn{7}{|c|}{2017} & \multicolumn{6}{|c|}{2018} \\
\hline & $6 / 19$ & $7 / 20$ & $8 / 24$ & $9 / 25$ & $10 / 26$ & $11 / 25$ & $12 / 24$ & $1 / 21$ & $2 / 24$ & $3 / 25$ & $4 / 26$ & $5 / 27$ & $6 / 25$ \\
\hline Time interval & & 31 & 35 & 32 & 31 & 30 & 29 & 28 & 34 & 29 & 32 & 31 & 29 \\
\hline Original observation data & & 22.9 & 25.5 & 21.5 & 18.8 & 16 & 14.4 & 13.5 & 15.5 & 12.6 & 14.3 & 12.4 & 11.5 \\
\hline Data with equal time-interval & & 22.8 & 22.5 & 20.8 & 18.7 & 16.5 & 15.4 & 14.9 & 14.1 & 13.4 & 13.8 & 12.4 & 12.3 \\
\hline
\end{tabular}

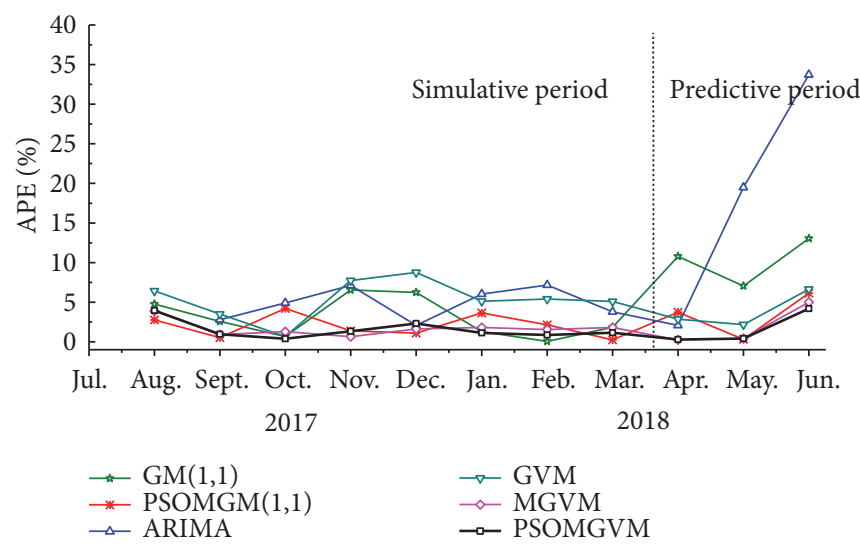

FIGURE 3: Comparison of the APE adopted the six competing models to forecast the settlement of high fill subgrade in Xiangli Expressway.

TABLE 3: Parameter values for the competing models.

\begin{tabular}{lcc}
\hline Parameters & $a$ & $b$ \\
\hline GM(1,1) & 0.0708 & 24.1432 \\
GVM & 0.0356 & -0.0013 \\
\hline
\end{tabular}

characteristics of huge amount of engineering, complex topographic and geological conditions, and difficult construction. The subgrade of Xiangli Expressway locates in the "V" shaped gully with the thickness of the subgrade fill up to $30 \mathrm{~m}$. The high fill subgrade of K34+480 segment was monitored from July 2017 to June 2018, and the observative results are shown in Table 2. According to the monitoring plan, it is monitored once every 2 to 10 days, depending on the construction progress, loading, weather conditions, and so on. The observative data was sorted out according to the month. The maximum time-interval is 35 days, the shortest time-interval is 27 days, and the data points with equal timeinterval are 30.91 days.

4.2. Simulation and Validation. In order to predict conveniently for further elaboration, the unequal data points should be reorganized by month. The methods to generate the data points with equal time-interval are elaborated in Section 3.1.1, as shown in Table 2. Then, the classical GM(1,1) and GVM models are calculated by the using of compiled Matlab program. The parameters $a$ and $b$ are obtained by the least square method, as shown in Table 3 . The simulative data points of the ARIMA model are acquired through the Eviews software, which can be referred to Agung [31]. The simulative and predictive values of competing models can be obtained based on the above methods, as shown in Table 4 .
With regard to the simulative values of the MGVM model, the relative errors are calculated by (16). Then, the state intervals of relative error are classified into three parts, namely, $S_{1}=[-5.02,-3.80), S_{2}=[-3.80,4.26)$, and $S_{3}=$ $[4.26,8.90]$. The occurrence numbers of $N_{1}, N_{2}$, and $N_{2}$ are 3,1 and 5, respectively. The one-step transition probability matrix is obtained through (19), namely,

$$
p=\left[\begin{array}{ccc}
0.5 & 0.5 & 0 \\
0 & 0 & 1 \\
0.2 & 0 & 0.8
\end{array}\right]
$$

The state probability of the simulative values can be calculated by (24), as shown in Table 4 .

According to this paper, the PSOMGVM prognostic model is applicated to predict the settlement of high fill subgrade. The parameter settings of the PSO algorithm are as follows. The number of particle group is 200, the maximum number of iterations iter $_{\max }$ is 500 , the particle velocity belongs to the interval of $[-0.5,0.5]$, and the particle position belongs to the interval of $[-5,5]$. The whitening weight coefficient of different states is obtained by the PSO algorithm. The whitening weight coefficients $\alpha_{1}, \alpha_{2}$, and $\alpha_{3}$ of different states are $0.4866,0.6917$, and 0.6389 , respectively.

For the GM(1,1), PSOMGM(1,1), ARIMA, GVM, MGVM, and PSOMGVM models, the minimum APE is $0.67,0.24$, 2.07, $0.67,0.24$, and 0.30 for the simulative period, and the maximum $A P E$ is $6.55,4.21,7.16,8.77,3.89$, and 3.95, respectively, as shown in Table 5. For the simulative and predictive periods, the minimum APE of the PSOMGVM model is the second lowest and second lowest, respectively. From Figure 3, it indicates that the APE line of PSOGVM 
TABLE 4: The comparison table of different prognostic models for settlement of high fill subgrade in Xiangli Expressway [unit: mm].

\begin{tabular}{ccccccccc}
\hline Year & Month & $X^{(0)}(k)$ & GM(1,1) & PSOMGM(1,1) & ARIMA & GVM & MGVM & PSOMGVM \\
\hline \multirow{4}{*}{2017} & Jul. & 22.90 & $/$ & $/$ & $/$ & $/$ & $/$ & 24.51 \\
& Aug. & 25.50 & 24.29 & 24.80 & $/$ & 23.86 & 24.49 \\
& Sept. & 21.50 & 20.95 & 21.39 & 22.10 & 20.75 & 21.31 & 21.29 \\
& Oct. & 18.80 & 18.93 & 18.01 & 19.72 & 18.93 & 18.56 & 18.73 \\
& Nov. & 16.00 & 17.05 & 16.22 & 17.14 & 17.24 & 16.10 & 16.21 \\
\multirow{2}{*}{2018} & Dec. & 14.40 & 15.30 & 14.56 & 14.70 & 15.66 & 14.63 & 14.73 \\
& Jan. & 13.50 & 13.67 & 13.01 & 12.69 & 14.19 & 13.26 & 13.35 \\
& Feb. & 15.50 & 15.51 & 15.83 & 14.39 & 16.34 & 15.26 & 15.37 \\
& Mar. & 12.60 & 12.37 & 12.63 & 12.12 & 13.24 & 12.37 & 12.46 \\
& Apr. & 14.30 & 12.76 & 13.77 & 14.00 & 13.89 & 14.27 \\
\hline \multirow{2}{*}{2018} & May & 12.40 & 11.53 & 12.44 & 14.82 & 12.13 & 12.35 & 14.26 \\
\hline
\end{tabular}

TABLE 5: The appraise indices of different prognostic models to predict the settlement of high fill subgrade in Xiangli Expressway.

\begin{tabular}{|c|c|c|c|c|c|c|c|c|}
\hline Year & Month & $X^{(0)}(k)$ & $\begin{array}{l}\text { GM(1,1) } \\
\text { APE (\%) }\end{array}$ & $\begin{array}{c}\text { PSOMGM(1,1) } \\
\text { APE (\%) }\end{array}$ & $\begin{array}{c}\text { ARIMA } \\
\text { APE } \\
(\%)\end{array}$ & $\begin{array}{l}\text { GVM } \\
\text { APE } \\
(\%)\end{array}$ & $\begin{array}{c}\text { MGVM } \\
\text { APE } \\
(\%)\end{array}$ & $\begin{array}{c}\text { PSOMGVM } \\
\text { APE (\%) }\end{array}$ \\
\hline \multirow{6}{*}{2017} & Jul. & 22.90 & 1 & 1 & 1 & 1 & 1 & 1 \\
\hline & Aug. & 25.50 & 4.76 & 2.76 & l & 6.42 & 3.89 & 3.95 \\
\hline & Sept. & 21.50 & 2.57 & 0.52 & 2.77 & 3.50 & 0.90 & 0.96 \\
\hline & Oct. & 18.80 & 0.67 & 4.21 & 4.89 & 0.67 & 1.29 & 0.39 \\
\hline & Nov. & 16.00 & 6.55 & 1.38 & 7.12 & 7.72 & 0.64 & 1.33 \\
\hline & Dec. & 14.40 & 6.25 & 1.09 & 2.08 & 8.77 & 1.61 & 2.31 \\
\hline \multirow{4}{*}{2018} & Jan. & 13.50 & 1.27 & 3.64 & 6.03 & 5.11 & 1.81 & 1.13 \\
\hline & Feb. & 15.50 & 0.05 & 2.15 & 7.16 & 5.40 & 1.54 & 0.86 \\
\hline & Mar. & 12.60 & 1.82 & 0.24 & 3.78 & 5.10 & 1.82 & 1.14 \\
\hline & Apr. & 14.30 & 10.79 & 3.73 & 2.07 & 2.86 & 0.24 & 0.30 \\
\hline \multicolumn{2}{|c|}{ MAPE (\%) } & & 3.86 & 2.19 & 4.49 & 5.06 & 1.52 & 1.37 \\
\hline \multicolumn{2}{|c|}{ RMSE } & & 0.83 & 0.44 & 0.77 & 0.95 & 0.38 & 0.38 \\
\hline \multirow{2}{*}{2018} & May & 12.40 & 7.04 & 0.31 & 19.50 & 2.17 & 0.43 & 0.41 \\
\hline & Jun. & 11.50 & 13.03 & 6.15 & 33.72 & 6.65 & 4.99 & 4.18 \\
\hline \multicolumn{2}{|c|}{ MAPE (\%) } & & 10.04 & 3.23 & 26.61 & 4.41 & 2.71 & 2.30 \\
\hline \multicolumn{2}{|c|}{ RMSE } & & 1.23 & 0.50 & 3.23 & 0.57 & 0.41 & 0.34 \\
\hline
\end{tabular}

Note: the optimal indices of the six competing models are in italic.

model is more close to the zero lines compared with the other competing models. Based on the APE analyses, it demonstrates that the PSOGVM model has a more excellent performance to predict the settlement of high fill subgrade compared with the other five competing models.

With regard to the appraise indices of the MAPE and RMSE, the minimum MAPE and RMSE are 1.37 and 0.38, respectively, both provided by PSOMGVM model for the simulative period. For the predictive period, the minimum $M A P E$ and RMSE are 2.30 and 0.34 , respectively, both calculated by the PSOMGVM model. Therefore, the accuracy and the ability of the PSOMGVM model outperform the other five competing models according to the MAPE and RMSE indices.
In addition, the predictive values of PSOMGVM model are not the same as the observative values to predict the settlement of high fill subgrade from Figure 4. Nevertheless, according to the appraise criteria of MAPE in Table 1, all the competing models, except for the ARIMA model, have excellent performance for the simulative and predictive periods.

4.3. Forecast. Through the analysis of Section 4.2, the performance of the PSOMGVM prognostic model outperforms the other five competing models, which is selected for forecasting the settlement of high fill subgrade. The predictive values for the settlement of high fill subgrade from July 2018 to December 2018 are shown in Table 6. 


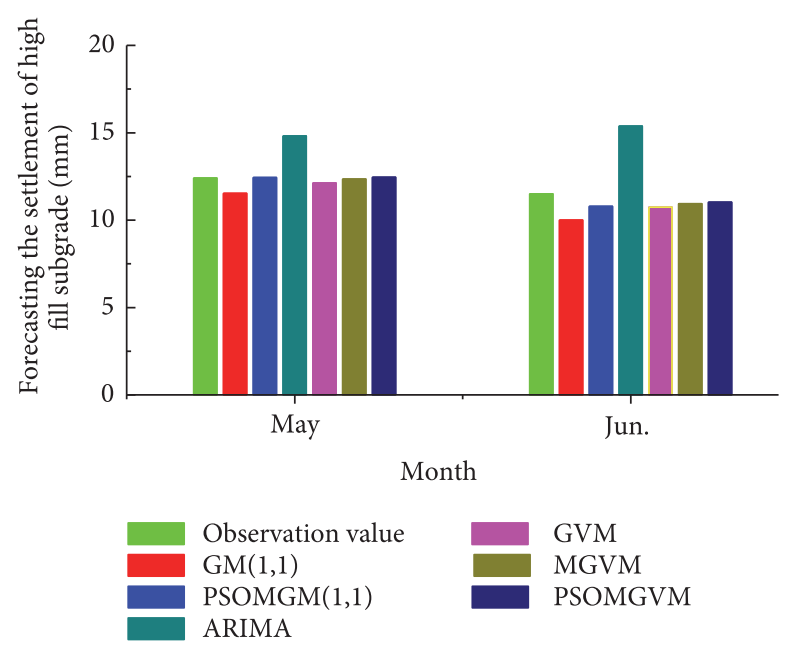

FIGURE 4: The observative and predictive settlement values of high fill subgrade in Xiangli Expressway using the competing models.

TABLE 6: The predictive settlement values of high fill subgrade using PSOMGVM prognostic model [unit: $\mathrm{mm}$ ].

\begin{tabular}{lcccccc}
\hline Year & \multicolumn{7}{c}{2018} \\
\hline Month & Jul. & Aug. & Sept. & Oct. & Nov. & Dec. \\
Predictive values & 10.77 & 10.23 & 9.72 & 9.25 & 8.79 & 8.37 \\
\hline
\end{tabular}

\section{Discussion}

As a result, we can draw a conclusion that the PSOMGVM model presented in this paper outperforms the other competing models to predict the settlement of high fill subgrade with higher prediction accuracy. From the GVM model to PSOMGVM model, we employed a gradually progressive optimization process and the comparison results indicated the proposed new model, called PSOMGVM model, based on PSO algorithm has a good fitting effect.

The grey prognostic model was suitable for time series with the feature of "small sample and poor information" [32]. The settlement of high fill subgrade not only has the grey characteristics of poor information and uncertainty, but also has the feature of nonlinearity and complexity [33]. The MGVM model could reflect the fluctuation law of sequence by determining the transfer law of sequence state, which could overcome the defect that the grey model cannot reflect the fluctuation of the data sequence and improve the prediction accuracy greatly. In order to efficiently search for the optimal whitening weight of the state interval, the improved PSO algorithm was introduced in this paper. This PSO algorithm has the advantages of fast searching speed and high efficiency. Nevertheless, it has the disadvantage of easily falling into local optimum, which is improved to a certain extent through the PSOMGVM algorithm proposed in this paper. In fact, to overcome the disadvantage of falling into local optimum, we had done more than ten times calculations and then chose the best whitening weight.
The modeling condition of the grey prognostic model is that the original sequence or construction data sequence by a variety of algorithms must have "quasi-exponential law." From the analysis of Section 2, it can be seen that the settlement of high fill foundation follows the characteristics of exponential law. However, the settlement of some high fill subgrade is not stable, and the grey model is inappropriate. With the passage of time, the grey level of the model will become larger and larger, and the long-term prediction effect of the grey prognostic model will not be well. Since the PSOMGVM model proposed in this paper is based on the Grey theory, it can only be suitable for short-term and medium-term prediction, and not appropriate for long-term prediction. In addition, the computational process of the model is more complex, which increases the application difficulty of prediction.

With regard to the research of the grey prognostic model, the following work can be carried out for further research. Firstly, based on the self-evolution characteristics of the original data sequence, the transformation method, which does not destroy the evolution law of the original sequence, will be studied. In order to realize the data mining and highlight of the original data sequence, we can combine data mining technology for further research. Secondly, the combination of the grey model and artificial intelligence is a vital method to solve uncertain system modeling. The traditional grey model has obvious linear feature. The artificial intelligence can further expand the model construction and relax the restrictions on parameter setting and model construction, which can make up for the shortcomings of the traditional grey model. Thirdly, in order to improve the long-term forecasting effect of the grey model, further research is needed to develop a novel grey prognostic model for longterm forecasting.

\section{Conclusion}

Based on the characteristics of the soil mechanics and the settlement monitoring data series of high fill subgrade, which has the characteristic of " $S$ " shape, the grey Verhulst model is applicated to predict the settlement of high fill subgrade. The PSOMGVM prognostic model based on the PSO algorithm and the Markov optimization is established. Through the theoretical analysis and practical verification, the following conclusions can be obtained.

(1) The settlement of high fill subgrade can be regarded as a grey system. The relationship between the land subsidence and the time conforms to the law of occurrence, development, maturity, and eventual extinction, which is described by the logistic equation. According to the unequal time-interval of settlement observation data, the grey Verhulst model with unequal time-interval is established.

(2) Combined with the grey theory, Markov chain, and PSO theory, the PSOMGVM prognostic model is established, which is applicated to the high fill subgrade of Xiangli Expressway in Yunnan Province. The results show that the average relative error of the PSOMGVM model is $1.03 \%$, which is better than $1.62 \%$ of the MGVM model and $4.08 \%$ of the GVM model. The PSOMGVM prognostic model has 
high prediction accuracy. It has significance for predicting postconstruction settlement and final settlement of high fill subgrade.

\section{Nomenclature}

GVM(1,1): $\quad$ Grey Verhulst model

MGVM: $\quad$ Markov grey Verhulst model

PSO: $\quad$ Particle swarm optimization

PSOMGVM: The grey Verhulst model based on PSO algorithm and Markov chain

GM: $\quad$ Grey model

PSOMGM(1,1): The grey model based on PSO algorithm and Markov chain

ARIMA: $\quad$ Autoregressive integrated moving average model

APE: $\quad$ Absolute percentage error

MAPE: $\quad$ Mean absolute percentage error

RMSE: $\quad$ Root mean squared error

$Y^{(0)}$ :

$X^{(0)}$ :

$X^{(1)}$ :

$n$ :

$Z^{(1)}$ :

$a:$

$b:$

$\widehat{x}^{(0)}$ :

$\widehat{x}_{M G V M}^{(0)}(i):$

$S_{i}^{u}, S_{k+1}^{u}$ :

$S_{i}^{l}, S_{k+1}^{l}$ :

$P_{i j}:$

$N_{i}$ :

$N_{i j}$ :

$\varepsilon(i)$ :

$P_{\text {best }}$ :

$g_{\text {best }}$ :

$V_{i}$ :

$X_{i}$ :

$x_{k d}$ :

$v_{k d}$ :

$h_{k d}$ :

$h_{\text {gd }}$ :

$r_{1}, r_{2}$ :

$c_{1}$ :

$c_{2}$ :
The raw data sequence with unequal time-interval

The data sequence with equal time-interval

Cumulative generating operator of $X^{(0)}$

Number of data in the sequence

The mean consecutive neighbors

operator

Development coefficient

Grey action coefficient

Predictive value of the grey Verhulst model

The predictive value of the time $i$ based on Markov chain

The upper limit of the $i$ state and the $k+1$ state

The lower limit of the $i$ state and the

$k+1$ state

The transition probability from state $S_{i}$ to state $S_{j}$

Occurrences number of state $S_{i}$

The transition number from state $S_{i}$ to state $S_{j}$

The relative error of data sequence $i$

The best position of the particles

Particle speeds

Particle positions

The position of the $k$ th particle

The speed of the $k$ th particle, which is

limited to the interval $\left(v_{\min }, v_{\max }\right)$

Historic optimal value of the $k$ th

particle

Historic optimal value of all particles in the search space

Pseudorandom number,

$r_{1} \in[0,1], r_{2} \in[0,1]$

Cognitive acceleration coefficient

Social acceleration coefficient
The global best position of the particles

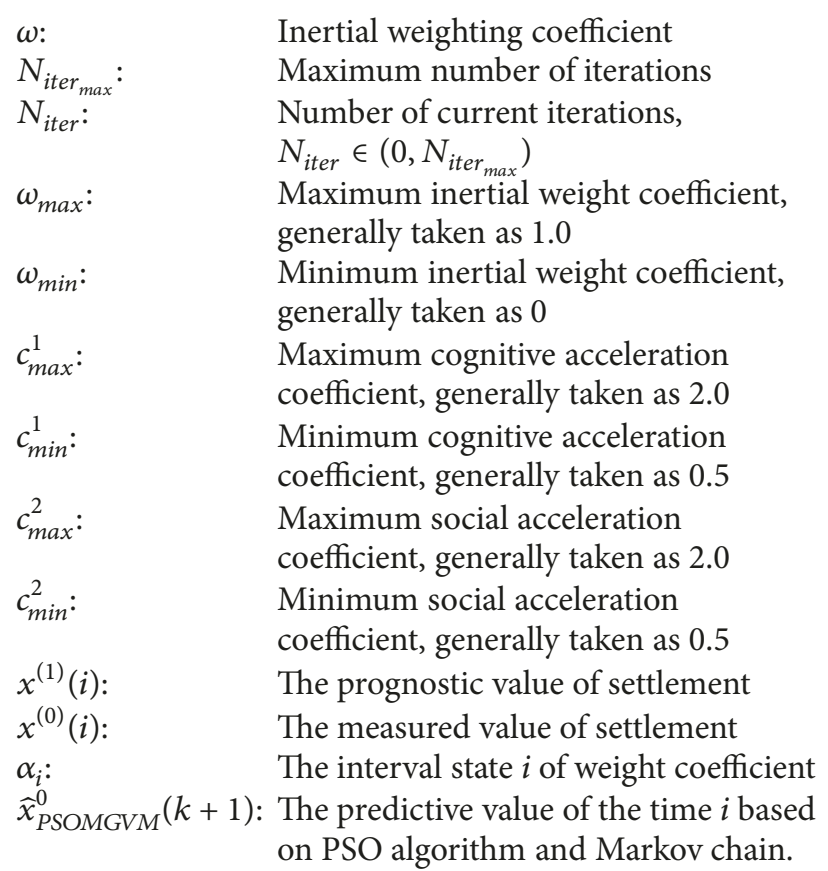

\section{Data Availability}

All data included in this study are available upon request by contact with the corresponding author or haiming0871@163.com

\section{Conflicts of Interest}

The authors declare that there are no conflicts of interest regarding the publication of this article.

\section{Acknowledgments}

This paper was supported by the National Natural Science Foundation of China (Grants nos. 51764020 and 51741410) and the National Key Research and Development Program of China (Project no. 2017YFC0804601). The authors would like to thank them for providing financial support for conducting this research.

\section{References}

[1] Y. Djonkamla, G. Dore, and J. P. Bilodeau, "Development of a prediction model of permanent deformation considering the physical properties of subgrade soil," Canadian Journal of Civil Engineering, vol. 43, no. 11, pp. 958-967, 2016.

[2] K. Terzaghi and R. B. Peck, Soil mechanics in engineering practice, John Wiley \& Sons, New York, NY, USA, 1967.

[3] M. Karakus and R. J. Fowell, "Effects of different tunnel face advance excavation on the settlement by FEM," Tunnelling and Underground Space Technology, vol. 18, no. 5, pp. 513-523, 2003.

[4] F. M. O. Mohamed, S. K. Vanapalli, and M. Saatcioglu, "Generalized Schmertmann equation for settlement estimation of shallow footings in saturated and unsaturated sands," Geomechanics and Engineering, vol. 5, no. 4, pp. 343-362, 2013. 
[5] D. M. Potts, "Numerical analysis: a virtual dream or practical reality? (42nd Rankine Lecture)," Géotechnique, vol. 53, no. 6 , pp. 535-573, 2003.

[6] A. Urzua, C. C. Ladd, and J. T. Christian, "New approach to analysis of consolidation data at early times," Journal of Geotechnical and Geoenvironmental Engineering, vol. 142, no. 10, 2016.

[7] W. He, G.-Y. Wang, and H.-H. Wang, "Nonlinear calculating method of pile settlement," Journal of Central South University of Technology, vol. 15, no. 2, pp. 63-68, 2008.

[8] Q. Huang, H.-W. Huang, B. Ye, D.-M. Zhang, L.-L. Gu, and F. Zhang, "Dynamic response and long-term settlement of a metro tunnel in saturated clay due to moving train load," Soils and Foundations, vol. 57, no. 6, pp. 1059-1075, 2017.

[9] G.-J. Huang, "New method to predict in advance settlement of ground under multi-stage filling," Chinese Journal of Geotechnical Engineering, vol. 29, no. 6, pp. 811-818, 2007.

[10] H. I. Park, K.-S. Kim, and H. Y. Kim, "Field performance of a genetic algorithm in the settlement prediction of a thick soft clay deposit in the southern part of the Korean peninsula," Engineering Geology, vol. 196, pp. 150-157, 2015.

[11] W. L. Liu, X. G. Wu, L. M. Zhang, J. Zheng, and J. Teng, "Global sensitivity analysis of tunnel-induced building movements by a precise metamodel," Journal of Computing in Civil Engineering, vol. 31, no. 2, 2017.

[12] N. Xu and Y.-G. Dang, "An optimized grey GM(2,1) model and forecasting of highway subgrade settlement," Mathematical Problems in Engineering, vol. 2015, Article ID 606707, 6 pages, 2015.

[13] C. Zhang, Z. Peng, and W. Peng, "Application of optimized grey discrete Verhulst model in settlement prediction of foundation pit," Journal of Central South University (Science and Technology), vol. 48, no. 11, pp. 3030-3036, 2017.

[14] J. Ming, Z. Fan, Z. Xie, Y. Jiang, and B. Zuo, "A modified grey verhulst model method to predict ultraviolet protection performance of aging B.mori silk fabric," Fibers and Polymers, vol. 14, no. 7, pp. 1179-1183, 2013.

[15] M. Evans, "An alternative approach to estimating the parameters of a generalised Grey Verhulst model: an application to steel intensity of use in the UK," Expert Systems with Applications, vol. 41, no. 4, pp. 1236-1244, 2014.

[16] B. A. Manivanna and A. Abudhahir, "Design of grey-verhulst sliding mode controller for antilock braking system," International Journal of Control, Automation, and Systems, vol. 14, no. 3, pp. 763-772, 2016.

[17] S. Miao, X. Hao, X. Guo, Z. Wang, and M. Liang, "Displacement and landslide forecast based on an improved version of Saito's method together with the Verhulst-Grey model," Arabian Journal of Geosciences, vol. 10, no. 3, pp. 51-60, 2017.

[18] Y. Wang, S. Yang, W. Qian, and X. Li, "Forecasting new product diffusion using grey time-delayed verhulst model," Journal of Applied Mathematics, vol. 2013, Article ID 625028, 6 pages, 2013.

[19] Y. Wang, Q. B. Song, S. MacDonell, M. Shepperd, and J. Y. Shen, "Integrate the $\operatorname{GM}(1,1)$ and verhulst models to predict software stage effort," IEEE Transactions on Systems, Man and Cybernetics-Part C: Applications and Reviews, vol. 39, no. 6, pp. 647-658, 2009.

[20] Z. X. Wang and Q. Li, "Modelling the nonlinear relationship between CO2 emissions and economic growth using a PSO algorithm-based grey Verhulst model," Journal of Cleaner Production, vol. 207, pp. 214-224, 2019.
[21] Y. Wang, L. Cheng, and L. T. Zhang, "Prediction of the mechanical behavior of a minicomposite based on grey Verhulst models," Ceramics - Silikaty, vol. 61, no. 4, pp. 372-377, 2017.

[22] X. Hu, Y. Wang, Y. Yu, D. Wang, and Y. Tian, "Research on the concentration prediction of nitrogen in red tide based on an optimal grey Verhulst model," Mathematical Problems in Engineering, vol. 2016, Article ID 9786107, 9 pages, 2016.

[23] B. Zeng, C. Li, G. Chen, and W. Zhang, "Verhulst model of interval grey number based on information decomposing and model combination," Journal of Applied Mathematics, vol. 2013, Article ID 472065, 8 pages, 2013.

[24] M. D. Braja, Advanced Soil Mechanics, McGraw-Hill, New York, NY, USA, 4th edition, 2013.

[25] L. Bjerrum, "Engineering geology of norwegian normallyconsolidated marine clays as related to settlements of buildings," Géotechnique, vol. 17, no. 2, pp. 83-118, 1967.

[26] N. A. Tsytovich and Y. K. Zaretsky, "The development of the theory of soil consolidation in the U.S.S.R., 1917-1967," Géotechnique, vol. 19, no. 3, pp. 357-375, 1969.

[27] W. Guo and J. Chu, "New observational method for prediction of one-dimensional consolidation settlement," Géotechnique, vol. 67, no. 6, pp. 1-7, 2017.

[28] W. Guo and J. Chu, "New observational method for prediction of one-dimensional consolidation settlement," Géotechnique, vol. 67, no. 6, pp. 516-522, 2017.

[29] J.-H. Yin and W.-Q. Feng, "A new simplified method and its verification for calculation of consolidation settlement of a clayey soil with creep," Canadian Geotechnical Journal, vol. 54, no. 3, pp. 333-347, 2017.

[30] T. E. Day and C. M. Lewis, "Forecasting futures market volatility," The Journal of Derivatives, no. 1, pp. 33-50, 1993.

[31] I. G. Agung, Advanced Time Series Data Analysis: Forecasting Using Eviews, John Wiley \& Sons, 2019.

[32] N. M. Xie and R. Z. Wang, "A historic review of grey forecasting models," The Journal of Grey System, vol. 54, no. 4, pp. 1-29, 2017.

[33] H. Yu, Y. Wang, C. Zou, P. Wang, and C. Yan, "Study on subgrade settlement characteristics after widening project of highway built on weak foundation," Arabian Journal for Science and Engineering, vol. 42, no. 9, pp. 3723-3732, 2017. 


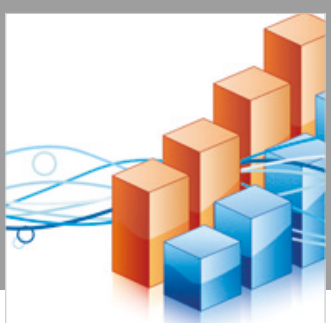

Advances in

Operations Research

\section{-n-m}
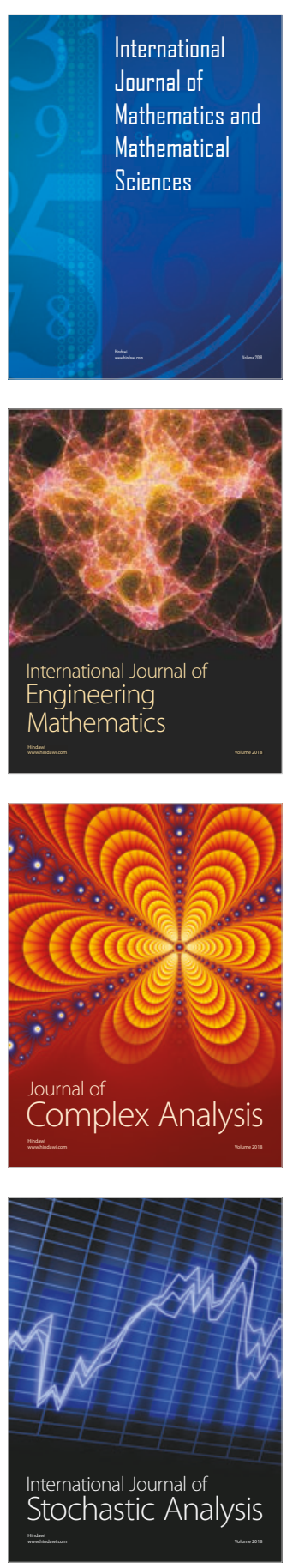
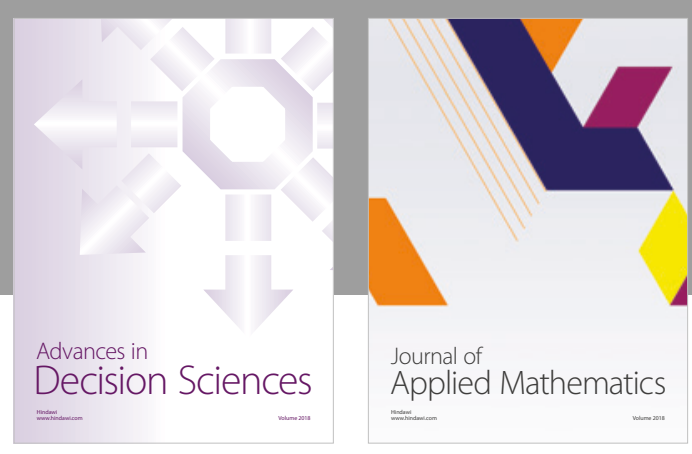

Journal of

Applied Mathematics
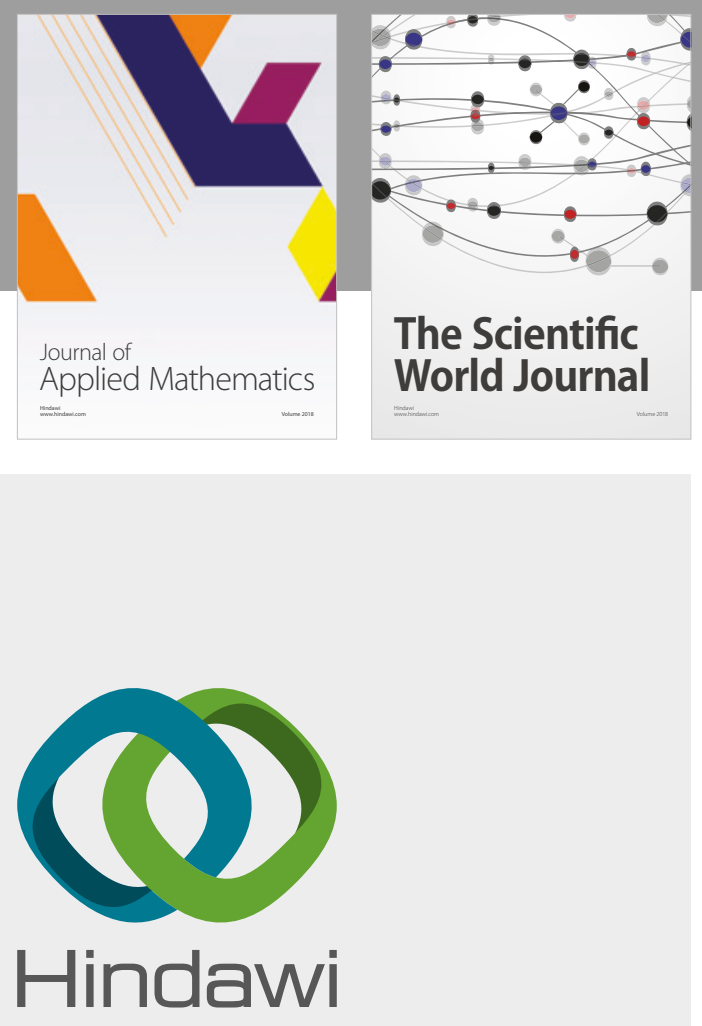

Submit your manuscripts at

www.hindawi.com

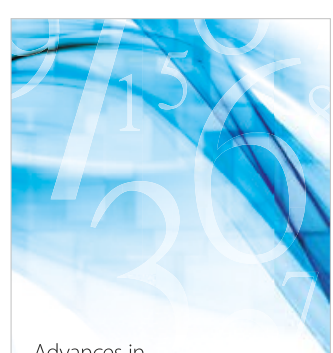

Advances in
Numerical Analysis
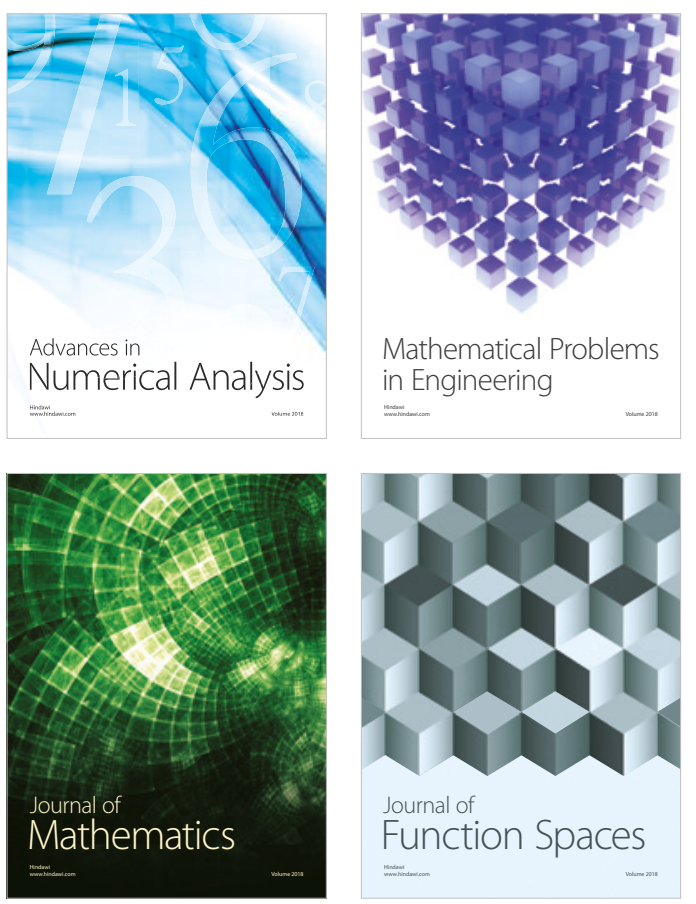

Mathematical Problems in Engineering

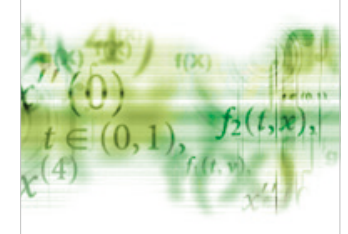

International Journal of

Differential Equations

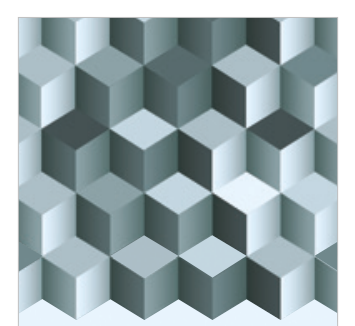

Journal of

Function Spaces

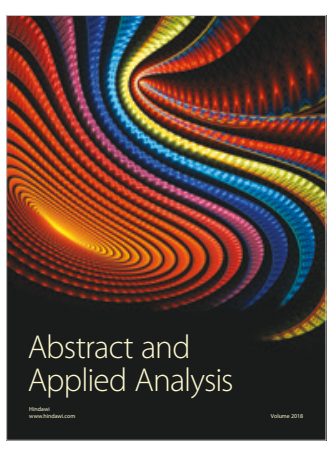

The Scientific

World Journal

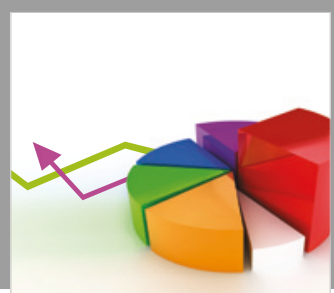

Journal of

Probability and Statistics
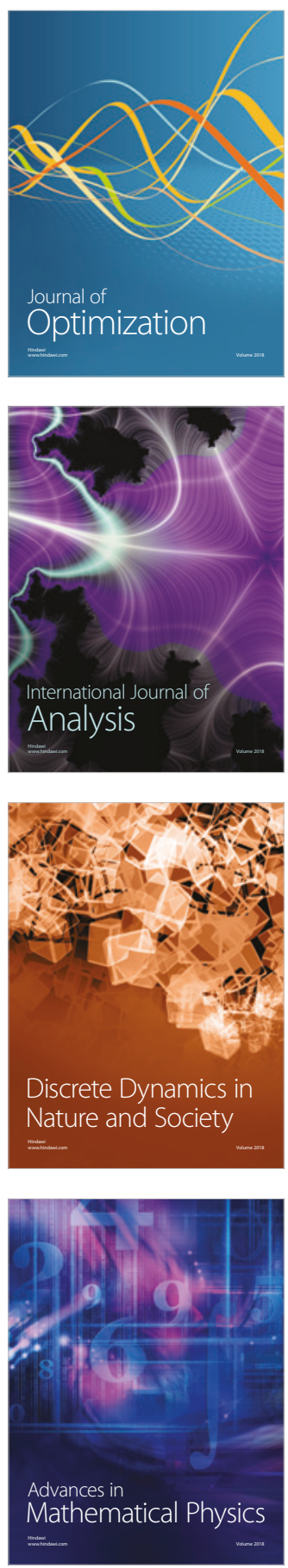